\title{
Frequency Dependence of Collisional Depolarization of Resonance Radiation
}

\author{
Frédéric Schuller and Wolfgang Behmenburg \\ (Z. Naturforsch. 30 a, 442-444 [1975]; received September 26, 1974)
}

\begin{abstract}
We have studied collisional depolarization of resonance radiation with special attention given to the line wings. It is shown that in the one particle quasistatic limit the fluorescence radiation is completely depolarized, independently of frequency and perturber density. By comparison with the integrated polarization degree it is concluded, that the polarization state of the radiation must exhibit a frequency dependence.
\end{abstract}

\section{Introduction}

The use of optical methods, in particular resonant scattering of light from atoms in gases or beams, for the study of atomic structure, collision processes and interactions involving excited states, has considerably increased in recent years. With respect to collisional depolarization of resonance radiation both theoretical and experimental studies have been concerned with phenomena like destruction of orientation and alignment or transfer of polarization of atomic systems by collisions ${ }^{1-4}$. These investigations have, however, been restricted to total cross sections, obtained from the scattered radiation energy integrated over the total spectrum of the line.

Differential cross sections for these processes, on the other hand, namely the dependence on the frequency of the scattered radiation, have to the authors' knowledge as yet not been considered. It should be pointed out in this connection, that conventional experiments on collisional line broadening are performed with unpolarized light, thus yielding no information on depolarizing processes. As in ordinary line broadening, the measurement of differential cross sections would yield much more information on atomic interactions and collision processes compared to measurements of total cross sections. In particular, detailed information on the splitting of the potential for the initial state of the optical transition (anisotropy of the forces) and nonadiabatic collisions may be obtained.

In this paper it will be shown for the first time, that a frequency dependence of the polarization state of the scattered radiation is to be expected.

Reprint requests to Dozent Dr. W. Behmenburg, Physikalisches Institut I, Universität Düsseldorf, D-4000 Düsseldorf, Universitätsstr. 1 .
The existence of the effect of frequency dependence of the polarization state may be qualitatively seen in the following manner. Quasistatically, the frequency $\omega$ of the radiation emitted from an atomic oscillator is related to its internuclear distance $R$ from a perturber through the difference of the potentials for the initial and final state of the line by $\hbar \omega(R)=V^{\mathrm{e}}(R)-V^{\mathrm{g}}(R) \equiv V(R)$. For simplicity we assume that $\omega$ increases with decreasing $R$. Since the impact parameter $b$ is by definition the smallest value $R$ during a collision, a correlation between $R$ and $b$ exists and thus a correlation between $\omega$ and $b$. Now, the criterium for a collision to be adiabatic with respect to the internuclear axis ${ }^{5}$ becomes increasingly fulfilled when $b$ decreases, assuming increasing splitting of the initial state potential with $R$. This means, that the angular momentum of the radiating atom will, with increasing probability, keep its orientation fixed with respect to the internuclear axis, but change it with respect to some space fixed axis, so that the emitted radiation becomes increasingly depolarized. Because of the correlation between $\omega$ and $b$ it follows from the above assumptions that the radiation becomes more and more depolarized with increasing $\omega$. Thus one would generally expect a change of the polarization state with frequency.

\section{Calculation of Integrated Fluorescence Intensities in the Impact Approximation}

We first calculate the fluorescence radiation intensities integrated over all frequencies.

We restrict ourselves to broad band excitation (halfwidth of the exciting line large compared to that of the fluorescence line) and sufficiently weak radiation fields, where effects like nonlinear absorption and stimulated emission may be neglected. 
Then the radiation flux corresponding to an electrical vector parallel to the unit vector $\boldsymbol{e}$ is given by the well known expression ${ }^{6}$

$$
\begin{aligned}
\Phi_{\text {etot }}= & \sum_{\substack{M_{\mathrm{e}} M_{\mathrm{e}^{\prime}} \\
M_{\mathrm{g}} \\
M M^{\prime}}} \varrho_{M_{\mathrm{e}} M_{\mathrm{e}}} \\
& \cdot(-1)^{M+M^{\prime}} e^{M}\left(\mu^{\mathrm{eg}}\right){ }_{M \mathrm{e}^{\prime} M_{\mathbf{g}}}^{-M} e^{M^{\prime}}\left(\mu^{\mathrm{ge}}\right) \bar{M}_{\mathbf{g}} \bar{M}_{\mathrm{e}}
\end{aligned}
$$

Here $g$ and $e$ refer to the ground and excited state respectively; the vectors $\boldsymbol{e}$ and $\mu$ are expressed in the spherical basis, all space quantum numbers being defined with respect to a space fixed frame.

In the following we consider the special case, that the electronic angular momentum quantum numbers associated with the ground- and excited state are $J_{\mathrm{g}}=0$ and $J_{\mathrm{e}}=1$ respectively, the nuclear spin being zero (example: transition $6{ }^{3} \mathrm{P}_{1} \rightarrow 6{ }^{1} \mathrm{~S}_{0}$ of the mercury ${ }^{198}$ line $\lambda 2537 \AA$ ). Furthermore we assume that the exciting radiation is linerly polarized along the $z$-direction.

Using the standard method of decomposition of the density matrix into irreducible tensor components ${ }^{6}$ the matrix elements $\varrho M_{\mathrm{e}} M_{\mathrm{e}^{\prime}}$ can be determined. It may be shown that no orientation appears and one obtains the following result:

$$
\begin{aligned}
& \varrho_{00}=K\left(\frac{1}{\Gamma+\gamma_{0}}+\frac{2}{\Gamma+\gamma_{2}}\right), \\
& \varrho_{11}=\varrho_{-1-1}=K\left(\frac{1}{\Gamma+\gamma_{0}}-\frac{1}{\Gamma+\gamma_{2}}\right),
\end{aligned}
$$

where $K$ is a constant. The quantities $\Gamma, \gamma_{0}$ are the relaxation rates of the scalar components of the density matrix due to radiation damping and quenching collisions respectively and $\gamma_{2}$ is the collisional relaxation rate of the alignment. We assume, that experimental conditions are such that $\gamma_{0}$ is zero.

We now consider two particular orientations of the vector $\boldsymbol{e}$, namely the directions parallel to the $z$ - and $x$-axis respectively of the space fixed frame; the corresponding components of $\boldsymbol{e}$ are given by $e^{M}=\delta_{M 0}$ and $e^{M}=-1 / \sqrt{2}\left(\delta_{M 1}-\delta_{M-1}\right)$ respectively. For the flux with respect to these directions one then obtains

$$
\begin{aligned}
& \Phi^{(z)}=\varrho_{00}\left(\mu^{\mathrm{eg}}\right)_{00}^{0}\left(\mu^{\mathrm{ge}}\right){ }_{00}^{0}, \\
& \Phi^{(x)}=-\frac{1}{2}\left[\varrho_{11}\left(\mu^{\mathrm{eg}}\right)_{10}^{1}\left(\mu^{\mathrm{ge}}\right)_{01}^{1}\right. \\
& \left.\quad+\varrho_{-1-1}\left(\mu^{\mathrm{eg}}\right)_{-10}^{-1}\left(\mu^{\mathrm{ge}}\right)_{0-1}^{1}\right] .
\end{aligned}
$$

We now define a quantity called integrated degree of polarization $P_{\text {tot }}$ by the relation

$$
P_{\mathrm{tot}}=\frac{\left(I_{\mathrm{tot}}\right)_{z}-\left(I_{\mathrm{tot}}\right)_{x}}{\left(I_{\mathrm{tot}}\right)_{z}+\left(I_{\mathrm{tot}}\right)_{x}}
$$

where the intensities $\left(I_{\mathrm{tot}}\right)_{z}$ and $\left(I_{\mathrm{tot}}\right)_{x}$ are proportional to the previously defined quantities $\Phi^{(z)}$ and $\Phi^{(x)}$ respectively. Remarking, that $\varrho_{11}=\varrho_{-1-1}$ we can show, that $P_{\text {tot }}$ reducés to

$$
P_{\text {tot }}=\left(\varrho_{00}-\varrho_{11}\right) /\left(\varrho_{00}+\varrho_{11}\right) \text {. }
$$

The formulas (3) and (5) are readily derived by using the Wigner-Eckart-Theorem.

For the purpose of discussion we express the density matrix elements by the relaxation rates using (2) with $\gamma_{0}=0$. We further introduce the cross section $Q_{2}$ for destruction of the alignment, defined $\gamma_{2}=n \bar{v} Q_{2}$ ( $\bar{v}$ and $n$ being the mean relative velocity of the colliding particles and the number density of the perturbers respectively). The total polarization degree then takes the form

$$
P_{\text {tot }}=\frac{1}{\left[1+\frac{2}{3} \frac{\gamma_{2}}{\Gamma}\right]}=\frac{1}{\left[1+\frac{2}{3} \frac{n \bar{v}}{\Gamma} \overline{Q_{2}}\right]} .
$$

We thus obtain the well known Stern-Vollmer type formula ${ }^{9}$.

\section{Fluorescence Intensities in the Quasistatic Wings of the Line}

In the far wings of the line the radiative flux at a certain frequency $\omega$ may be computed on the basis of the quasistatic one particle approximation. Furthermore we may assume that the atom is in an adiabatic state with respect to the internuclear axis, i. e. for a given value of the space quantum number with respect to that axis we associate a well defined binary potential curve. Under these assumptions the radiative flux parallel to $\boldsymbol{e}$ may be written as follows:

$$
\begin{gathered}
\Phi_{\boldsymbol{e}}(\omega)=C \varrho \sum_{\substack{M_{\mathrm{e}} \\
M M^{\prime}}}(-1)^{M+M^{\prime}} \boldsymbol{e}^{M}\left(\mu^{\mathrm{e} g}\right)-{ }_{M \mathrm{e} 0}^{M} \\
\cdot e^{M^{\prime}}\left(\mu^{\mathrm{ge}}\right)-M^{\prime} \delta\left[\omega-\omega_{M \mathrm{e}}(R)\right] \mathrm{d} \boldsymbol{R} .
\end{gathered}
$$

Here all space quantum numbers refer to the internuclear axis and $C$ and $\varrho$ are constants. The frequency $\omega_{M_{\mathrm{e}}}(R)$ is given by the difference potential according to the relation

$$
\omega_{M \mathrm{e}}(R)=\frac{1}{\hbar} V_{M \mathrm{e}}(R)
$$

where $R$ is the internuclear distance.

In establishing the formula (7) we assumed that the density matrix with respect to the internuclear 
axis is of the form $\varrho M_{\mathrm{e}} M_{\mathrm{e}^{\prime}}=\varrho \delta_{M_{\mathrm{e}} M_{\mathrm{e}^{\prime}}}$, resulting from the fact, that all orientations of that axis in space are equally probable.

As in the previous paragraph we now consider two particular directions of $\boldsymbol{e}$, parallel to the $z$ and $x$-axis respectively of a space fixed system. Components of $\boldsymbol{e}$ are then given by $e^{M}=D_{0 M}^{1}$ for $\boldsymbol{e}$ parallel $z$ and $e^{M}=1 / \sqrt{2}\left(D_{-1 M^{-}}^{1} D_{1 M}^{1}\right)$ for $\boldsymbol{e}$ parallel $x ; D^{1}$ is a rotation matrix depending on the Euler angels which specify the orientation of the internuclear axis with respect to the space fixed frame.

We now calculate the flux with respect to the two directions of $\boldsymbol{e}$. After averaging the products of $D^{1}$. matrix elements over all Euler angels we obtain the following result:

$$
\begin{aligned}
\overline{\Phi^{(z)}(\omega)} & =\frac{8 \pi^{2}}{3} C \varrho \sum_{M_{\mathrm{e}}}(-1)^{M_{\mathrm{e}}}\left(\mu^{\mathrm{eg}}\right)_{M \mathrm{e} 0}^{M \mathrm{e}}\left(\mu^{\mathrm{ge}}\right)_{0 M \mathrm{e}}^{-M \mathrm{e}} \\
& \cdot \delta\left[\omega-\omega_{M \mathrm{e}}(R)\right] R^{2} \mathrm{~d} R=\overline{\Phi^{(x)}}(\omega)
\end{aligned}
$$

Introducing the intensities $I_{z}(\omega) \sim \overline{\Phi^{(z)}(\omega)}$ and $I_{x}(\omega) \sim \overline{\Phi^{(x)}(\omega)}$ and defining a differential polarization degree

$$
P(\omega)=\left[I_{z}(\omega)-I_{x}(\omega)\right] /\left[I_{z}(\omega)+I_{x}(\omega)\right]
$$

we thus prove, that in the far wings of the line $P(\omega) \equiv 0$, i. e. the fluorescence radiation is completely depolarized.

\section{Discussion}

From the result expressed by (6) it is seen, that in general the integrated polarization degree has a

1 W. Hanle, Z. Physik 41, 164 [1927].

2 E. E. Nikitin, J. Chem. Phys. 43, 744 [1965].

3 M. Elbel and W. B. Schneider, Z. Physik 241, 244 [1971].

4 J. Guiry and L. Krause, Phys. Rev. A 6, 273 [1972] finite value, which decreases with increasing perturber density. For $n \rightarrow \infty$ one has $P_{\text {tot }}=0$, a result which is in agreement with intuition and experiment. On the other hand we have shown that at a given frequency in the quasistatic wings of the line there should be complete depolarization of fluorescence radiation at any density. From this we have to conclude, that there is a dependence of the polarization degree on frequency. Moreover one may expect, that at a given perturber density the central region of the line profile is stronger polarized than the line wings. These conclusions are in line with the qualitative arguments given in the introduction.

Strictly speaking the derivation of formula (6) for the total polarization degree is based on the impact approximation, which is valid only in the line core. Since, however, the latter yields the main contribution to the total line intensity, the general situation should not be affected by this restrictive remark.

Our approach does not allow us to obtain more detailed information on the frequency dependence of the polarization degree. Thus it seems highly desirable to develope a theory based on a general formalism.

The authors take pleasure in acknowledging the financial support of the "Gesellschaft der Freunde und Förderer der Universität Düsseldorf" during the preparation of the manuscript.

5 F. Schuller and B. Oksengorn, J. Physique 30, 531 [1969].

${ }^{6}$ R. Seiwert in Erg. Exakt. Naturwiss. 41, 143 [1968].

7 A. C. G. Mitchell and M. W. Zemansky, Resonance Radiation and Excited Atoms (Cambridge U.P., Cambridge, England 1934). 